\title{
BIOMASS End-to-End Mission Performance Simulator
}

\author{
Paco López-Dekker *, Francesco De Zan*, Thomas Börner*, Marwan Younis*, \\ Kostas Papathanassiou*, Tomás Guardabrazo ${ }^{\dagger}$, Valerie Bourlon ${ }^{\ddagger}$, Sophie Ramongassie ${ }^{\ddagger}$, Nicolas Taveneau, \\ Lars Ulander $^{\S}$, Daniel Murdin ${ }^{\S}$, Neil Rogers $₫$, Shaun Quegan $₫$ and Raffaella Francol \\ * Microwaves and Radar Institute, German Aerospace Center (DLR), Oberpfaffenhofen, Germany \\ $\dagger$ Deimos Space $\ddagger$ Thales Alenia Space $\S$ Swedish Defence Research Agency (FOI) \\ ฯ University of Sheffield II European Space Agency (ESA)
}

\begin{abstract}
This paper discusses the implementation of an endto-end simulator for the BIOMASS mission. An overview of the system architecture is provided along with a functional description of the modules that comprise the simulator.
\end{abstract}

\section{INTRODUCTION}

Biomass is a biophysical property of vegetation that relates to the amount of carbon stored in the terrestrial environment. While the world's forests contain the largest proportion of carbon in living vegetation global and accurate quantification of stock and dynamics - occurring as a consequence of, for example, deforestation, regrowth, management or fires remains a significant but pressing challenge.

This uncertainty remains because of the lack of a systematic and reliable mechanism for differentiating biomass levels across large areas. In this context, European scientists proposed in the frame of ESA's 7th Earth Explorer [1] program BIOMASS[2], [3], a mission with the objective to reduce the uncertainty in the worldwide spatial distribution and dynamics of forests leading to improved present assessments and future projections of the carbon cycle.

BIOMASS is based on a P-band Synthetic Aperture Radar that will systematically acquire fully- (quad-) polarized image data in an interferometric mode over all major forested areas on the globe. The inversion methodology is then based on backscatter intensity measurements at different polarizations and interferometric coherence measurements at different polarizations.

BIOMASS is currently in the Phase-A stage, in parallel with the two other competing mission proposals: $\mathrm{CoReH}_{2} \mathrm{O}$ and Premier. A common critical key element for the final selection procedure is an End-to-End mission performance assessment, for which a End-to-End Simulator (E2ES) tool is being implemented. This BIOMASS E2ES (BEES) needs to go beyond the straightforward (but fundamental) radiometric sensitivity analysis and include disturbances or errors due to:

- The radiometric accuracy and stability, and radiometric biases.

- Ionospheric artifacts: Faraday rotation and scintillation effects.

- The effect of range and azimuth ambiguities.

- Phase stability (within an acquisition but also within channels or within repeat pass acquisitions)
- Channel imbalance and cross talk.

- Decorrelation sources: temporal and spectral shift.

The End-to-End nature of this tools means that the starting point of the simulation are maps providing a (simplified) geophysical descriptions of forest scenes, for which the main parameter of interest is the biomass (in mass per surface unit). Likewise, the final output of the tool are the estimates of this biomass parameter.

\section{END-TO-End Simulator ARCHITECTURE}

This section describes the architecture of BEES, which is illustrated in Fig. 1. The modular structure of the E2ES corresponds not only to a logical breakdown of the simulation process, but reflects also the fact that the most of the modules are being developed by different research groups in the context of specific studies. To streamline the integration of these heterogeneous modules into a single tool, the E2ES is built using ESA's Open Simulation Framework (OpenSF) [4]

An important architectural aspect of BEES is the attempt to find a balance between physical fidelity to the system being simulated, and computational efficiency.

These modules are briefly discussed in the following subsections.

\section{A. Geometry Module}

This module calculates the common geometric relations needed by all the other modules. Basically, on a common ground range grid, and for all simulated acquisitions, it calculates the slant range, look and incidence angle, the cross-track interferometric baseline (expressed as $k_{z}=2 \pi / h_{a m b}$ ), etc.

\section{B. Observing System Simulator (OSS)}

It characterizes the simulated BIOMASS SAR system. This module is divided in two independent modules:

IRF Module: This sub-module provides the end-to-end impulse response function (IRF) of the system considering the sensor physical characteristics (antenna pattern, orbit height, pulse bandwidth, etc.) and the SAR processing (processed Doppler bandwidth, range/azimuth windowing used, etc.).

The use of the end-to-end IRF is a key aspect of the E2ES, as it avoids generating raw data, which is computationally very costly, in particular at P-band due to the very long synthetic 


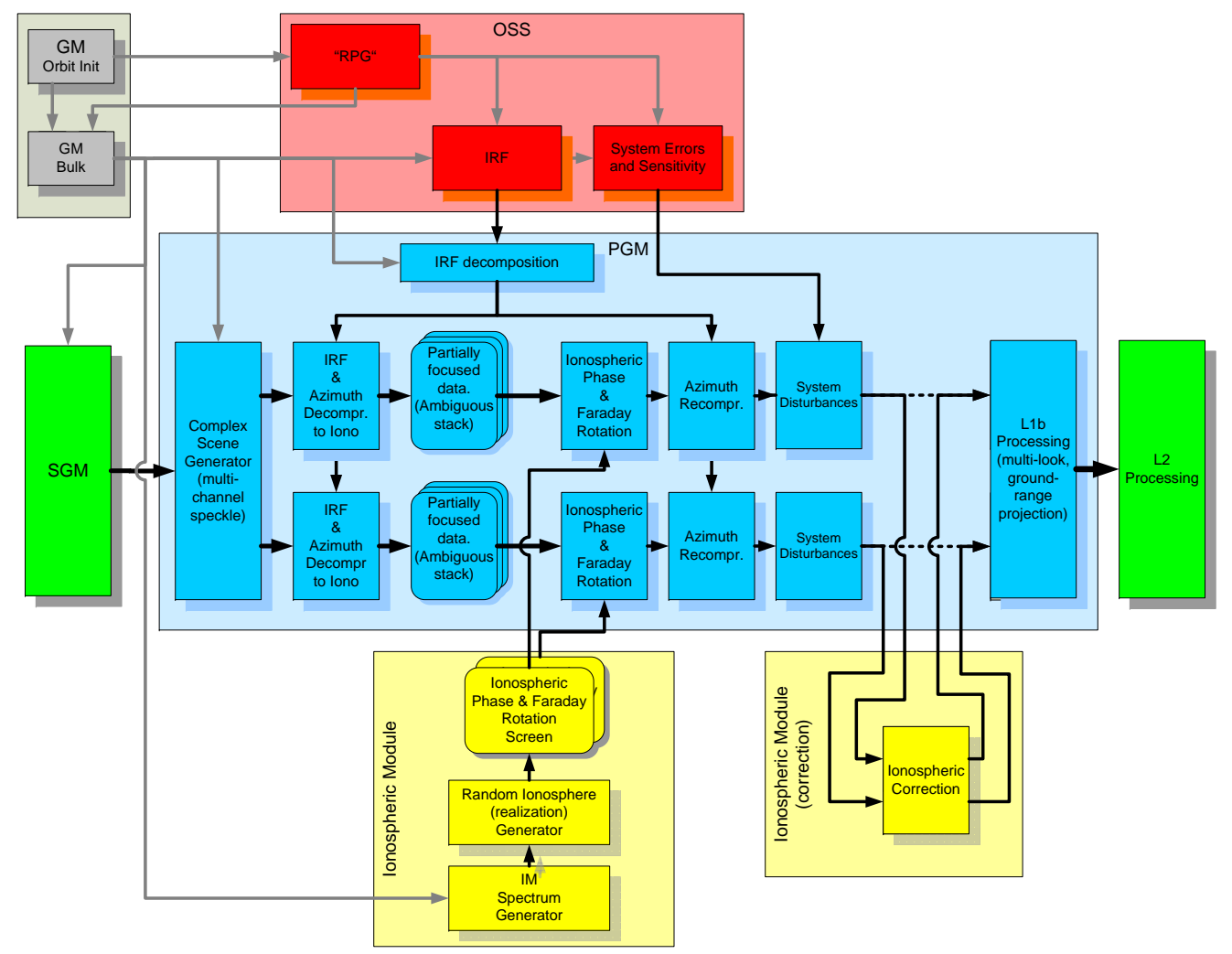

Fig. 1: Block diagram of the End-to-End simulator

aperture. Likewise, it avoids the equally computationally intensive SAR processing step.

It is worth emphasizing that the IRF partially represents the radar and the processing chain at the ground segment. A natural consequence of this approach is that the errors and disturbances introduces during the simulation should be residual (post-calibration) ones, since the ground-segment will apply all known calibration factors to the data as part of the standard processing.

System Error and Sensitivity (SES): The SES sub-module returns the different parameters describing the performance of the system:

- Noise equivalent $\sigma_{0}$ (NESZ)

- Range and azimuth ambiguity to signal ratios. In addition, an ambiguity mapping is provided, giving the location and relative amplitude of the individual ambiguities.

- Phase and amplitude stability (long and short term).

- Residual channel imbalance and cross-talk.

- Localization accuracy.

These values are calculated as a function of range.

Two different OSSs have been developed to simulate the two different Phase-A designs.

\section{Scene Generation Module (SGM)}

This module provides the scene as a map of the second order statistics of the scene, tying a specific extended covariance matrix to each position on the ground. The simulated forest is described by a forest type (currently Tropical and Boreal forests are considered), a mean biomass level, and a gradient. As a first step, the SGM generated $1 \mathrm{~km}^{2}$ patches of forest with uniform biomass levels. Within each patch, the mean biomass level is converted to a tree-dimension distribution (Weibull or exponential). Using this distribution, a random collection of trees with different diameters and heights is generated. These trees are then clustered to form either a uniform tree distribution (typical of Boreal forests) or a clumpy one (associated to Tropical forests). The resulting forest patches are then converted to a $10 \times 10$ grid giving the biomass level and tree height (expressed as H100, the mean height of the 100 tallest trees per hectare) in a 1 ha square.

These 1 ha averaged biomass levels and tree-heights are then passed to a forward model derived from experimental data. As additional input parameters this model requires the nominal incidence angle, the vertical wavenumber $\left(k_{z}\right)$, the temporal baseline between acquisitions and the biome id. Optionally, 
the model can also ingest a digital elevation model (DEM).

\section{Ionosphere Modules}

BEES incorporates an ionosphere generation module (IGM), which simulates ionospheric disturbances, and an ionospheric correction module (ICM). The IGM provides a map of Faraday rotation (FR) across the SAR image based on the satellite and radar geometry, a model of total electron content (TEC) along the propagation path and a model geomagnetic field. The IGM also simulates phase fluctuations due to ionospheric scintillation (the process of scattering from electron density irregularities in the ionosphere). This is provided as a twodimensional thin phase screen, pseudo-randomly generated from a database of spatial phase spectrum parameters derived from the WBMOD model [5] for a variety of geomagnetic activity indices and over a solar cycle (1995-2005).

The ICM provides interfaces to a range of FR estimation and correction algorithms, which estimate the FR angle from the covariance between polarization channels but with an ambiguity of typically $+/-n$ pi/2, where $n$ is an unknown integer. A model estimate of FR is therefore used to resolve the ambiguity in the FR estimates. The ICM will also estimate and correct the azimuthal shift in multiple-acquisition images by means of either an intensity correlation analysis for subwindows within the image, and/or by determining the difference in TEC gradient and subsequent azimuth shift from the difference in FR estimates (between slave and master image) using a model geomagnetic field estimate.

Further details of both ionospheric modules are provided in [6].

\section{E. Product Generation Module (PGM)}

The PGM is the heart of BEES, as it is the connecting node of all the other modules. It generates simulated SAR from the scene provided by the SGM, using the system characterization provided by the OSS and including (if so required) the IGM generated ionospheric distortions. An important aspect of the PGM is the capability to select which subset of disturbing phenomena are included in the simulation. The module is divided in two sub-modules:

PGM-L1a: generates coregistered multi-channel SLC SAR images without the Ionospheric Correction.

PGM-L1b: performs the multi-looking operation (estimation of the covariance matrices) and projection to ground range.

The PGM-L1a performs the following steps:

1) Calculates the Cholesky decomposition of the input covariance matrices.

2) Generates correlated multi-channel noise, by multiplying vectors of independent randomly distributed jointly Gaussian complex random vectors by coloring matrix obtained from the previous step. The resulting data is over-sampled w.r.t. the final SLC resolution.

3) Applies the interferometric phase to the interferometric data-pairs. This implicitly introduces a spectral shift in the image, which will cause a coherence loss.
4) Convolution with the OSS-generated IRF. In general, this is a range-dependent function.

5) The images are decompressed in azimuth to Ionospheric height where the ionospheric effects are introduced (for the equivalent SAR system flying at ionospheric height, the ionospheric distortion can be applied directly pixel by pixel to the range compressed data).

6) Interferometric phase flattening (removal of flat Earth phase).

7) Introduction of range and azimuth ambiguities. This implies adding a number of ambiguous images tot the main image. Each ambiguity is generated following the previous steps.

8) Introduction of system disturbances: random noise, phase and amplitude errors due to long and short term system stability, polarimetric channel imbalance and cross-talk. These errors are considered post-calibration residual errors.

The result of these steps is a set of SLC images typically representing the in total 8 channels of a PolInSAR acquisition. These images are then passed to the ICM, which returns an equivalent set of ionospheric-corrected SLCs, which are the input to the PGM-L1b sub-module. The PGM-L1b module estimates the extended covariance matrices from the data by applying a Gaussian spatial multi-looking window to all the co- and cross-channel products.

\section{F. $L 2$ retrieval module (L2RM)}

This implements the inversion algorithm, producing biomass and forest heights estimates from the PGM supplied L1b data. The L2RM produces two independent biomass estimates. The first is derived from the HV (cross-polar) backscatter coefficient, using the empirical Above-Ground Dry Biomass (AGDB) to $\sigma_{0}$ relations derived during the BioSAR- 1 campaign. The second estimate is generated from the PolInSAR data inverting a Random Volume over Ground (RVoG) model to obtain a H100 estimate and applying the H100-to-biomass allometric relations derived during BioSAR.

The two estimates are combined using a Bayesian MMSE approach.

\section{G. Performance Evaluation Module}

In addition to the previously described modules, which comprise the actual E2ES. An extra module is being developed to automate the performance analysis by comparing the biomass maps generated by the SGM with the biomass estimates produced at the end of the simulation chain.

\section{IMPLEMENTATION}

As discussed, the overall system is implemented using the OpenSF framework. OpenSF provides a Human-machine interface (HMI) to set-up the simulator out of a collection of OpenSF-compliant modules, and also to configure a particular run of the simulator by editing the configuration parameters for each module (physically these are stored as xml files). It also runs the simulation, keeping track of the simulation status 

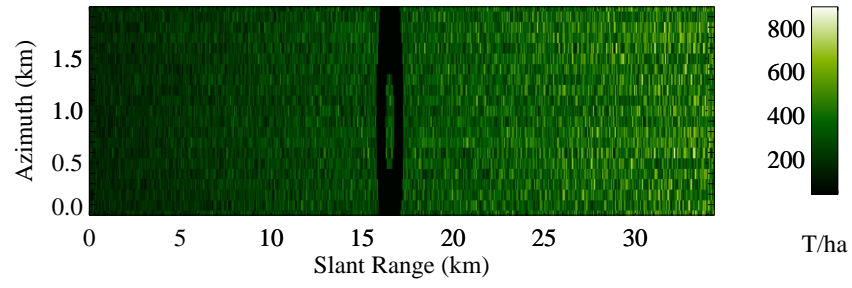

(a) Biomass

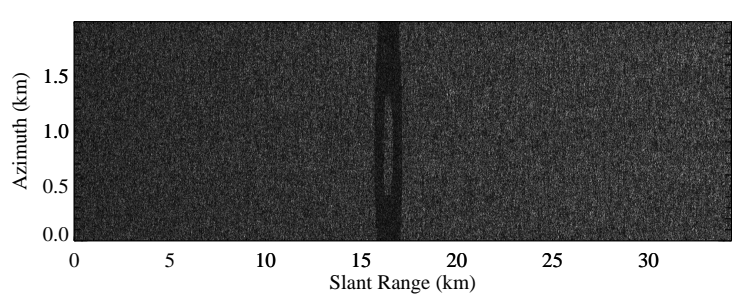

(b) SLC (HH)
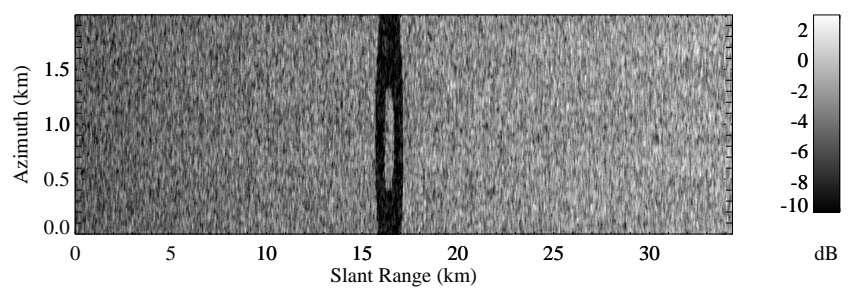

(c) NRCS $(\mathrm{HH})$

Fig. 2: From top to bottom: example of a SGM generated biomass map; amplitude of the single-look HH-channel SAR image; estimated normalized radar cross-section.

and error reporting. In addition, OpenSF allows configuring and executing a batch of simulation, optionally varying some parameters according to some pre-defined rule. This feature can be used, for example, for perturbation analysis or, as in the case of BEES, to generate multiple random realizations of a given simulation in a Monte Carlo approach.

The individual modules are developed in an heterogeneous combination of several programming languages and environments (mainly C, MATLAB and IDL). The entire simulator is designed to run on a standard Linux work-station.

Although at time of writing BEES is not fully implemented, a preliminary computational performance analysis has been made. Currently, the simulation of a $200 \mathrm{~km}^{2}$ scene requires in the order of 200 seconds simulation time. With the missing features included, this computation time is expected to stay under 300 seconds, thus allowing running hundreds of Monte Carlo repetitions in a time span of hours.

\section{RESUlTS}

Figure 1 shows an example of some products generated by BEES. Fig. 2(a) shows a SGM generated biomass map pro-

\begin{tabular}{|c|c|c|c|}
\hline Index & Distribution & Clark/Evan Index & Notes \\
\hline 1 & Weibull & 1.8 & Boreal Forest \\
2 & Weibull & 0.8 & No ecosystem \\
3 & Exponential & 1.8 & No ecosystem \\
4 & Expoinential & 0.8 & Tropical Forest \\
\hline
\end{tabular}

TABLE I: Forest types defined for reference scenarios

jected on a slant-range-azimuth grid. The image corresponds to a $70 \mathrm{~km}$ ground range (more or less the total swath envisioned for BIOMASS) by $2 \mathrm{~km}$ azimuth scene. A scene with a smooth biomass gradient of $4 \mathrm{~T} / \mathrm{ha} / \mathrm{km}$ in ground range and $1 \mathrm{~T} / \mathrm{ha} / \mathrm{km}$ in azimuth was simulated. In addition, a low biomass ring was introduced in center of the scene in order to have a distinct feature in the image. The fine granularity of the image reflects the simulated local heterogeneity of the forest.

Fig. 2(b) shows the amplitude of the simulated SLC (HH channel) as generated by the PGM. Aside from the low biomass ring, the most salient feature is, as expected, the high frequency granularity due to speckle. Fig. 2(c) shows the estimated NRCS estimated applying a multi-look factor of 20. Some of the random features in the original biomass map as well as the mean gradient can be identified in the NRCS image.

\section{OUTLOOK}

BEES is currently in its latest development stages and is scheduled to be completed during Summer 2011. Once completed, it will be used to evaluate the expected performance of BIOMASS for the two alternative SAR system designs proposed during the Phase-A study. To perform this evaluation a set of reference scenarios have been defined, corresponding to 4 forest types (see Table I) and a range of average biomass levels.

For each scenario, the system will be evaluated at two levels:

1) The fidelity L1b products will be evaluated by comparing the elements of the covariance matrices generated by the SGM to those estimated by the PGM-L1b module.

2) The end-to-end performance of the mission will be assessed by comparing the biomass an $\mathrm{H} 100$ tree height generated by the SGM and estimated by the L2RM.

\section{REFERENCES}

[1] J. Bezy, P. Bensi, C. Lin, Y. Durand, F. Heliere, A. Regan, P. Ingmann, J. Langen, M. Berger, M. Davidson, and H. Rebhan, "ESA future earth observation explorer missions," in Geoscience and Remote Sensing Symposium, 2007. IGARSS 2007. IEEE International, 2007, pp. 212-215.

[2] "BIOMASS - to observe global forest biomass for a better understanding of the carbon cycle, report for assessment," ESA, Tech. Rep. ESA SP1313/2, Nov. 2008.

[3] F. Heliere, C. Lin, F. Fois, M. Davidson, A. Thompson, and P. Bensi, "BIOMASS: a p-band SAR earth explorer core mission candidate," in Radar Conference, 2009 IEEE, 2009, pp. 1-6.

[4] "OpenSFwiki," http://opensf.deimos-space.com/. [Online]. Available: http://opensf.deimos-space.com/

[5] J. A. Secan, R. M. Bussey, E. J. Fremouw, and S. Basu, "Highlatitude upgrade to the wideband ionospheric scintillation model," Radio Science, vol. 32, no. 4, pp. PP. 1567-1574, 1997. [Online]. Available: http://www.agu.org/journals/ABS/1997/97RS00453.shtml

[6] N. Rogers and S. Quegan, "Simulation of ionospheric effects and their mitigation for the ESA BIOMASS p band Space-Based radar," 2011. 